Rapid Reviews COVID-19

\title{
Review 1: "Single-Molecule Dynamics of SARS-CoV-2 5' Cap Recognition by Human elF4F"
}

Published on: Jul 21, 2021

License: Creative Commons Attribution 4.0 International License (CC-BY 4.0). 


\section{$\underline{\text { RR:C19 Evidence Scale rating by reviewer: }}$}

- Reliable. The main study claims are generally justified by its methods and data. The results and conclusions are likely to be similar to the hypothetical ideal study. There are some minor caveats or limitations, but they would/do not change the major claims of the study. The study provides sufficient strength of evidence on its own that its main claims should be considered actionable, with some room for future revision.

$* * * * * * * * * * * * * * * * * * * * * * * * * * * * * * * * * * * * * * * *$

\section{Review:}

Although the subject (COVID-19 mRNA) is of extreme interest due to the current worldwide pandemic, this reviewer does not feel that there is sufficiently new material presented to justify publication - even though many of the experiments appear to have been done well and with controls. Other laboratories have examined many of the interactions of the eIF4F subunits with mRNA and this is acknowledged in this article. In addition, several concerns about the experimental methodology are raised.

\section{Concerns:}

1. Regarding the fluorescently-labeled eIF4E, it is not clear if the labeling is random (to more than one site on eIF4E or targeted to a specific amino acid) and whether this might influence results. Secondly, if about $50 \%$ is labeled, does the unmodified eIF4E serve as a "competitive" inhibitor?

2. Regarding the eIF4A ATPase assay, do the authors know that the rate of oxidation of NADH is much faster than the possible rate of ATP hydrolysis?

3. In the results, the authors wrote: "Thus, the viral untranslated regions support eIF4F-dependent translation". Is this true for both UTRs or just the 5' UTR?

4. For experiments with more than a single subunit, do the authors have proof of the degree with which complexes are formed (i.e. eIF4G + eIF4E $\rightarrow>$ eIF4G•4E)?

Secondly, what are the kinetics for the formation of complexes, especially eIF4F, when individual subunits are added together for the assay?

5. The authors used proteins expressed individually, although the eIF4G is not fulllength. How does the observed eIF4F activity compare to native eIF4F? 
6. For the presumed stem-loops in the COVID-19 5' UTR, what is the calculated $\Delta \mathrm{G}$ of the stems? 